\title{
Electrical Stimulation Therapy for Gastroesophageal Reflux Disease
}

\author{
Nicolaas F Rinsma, ${ }^{1}$ Nicole D Bouvy, ${ }^{2}$ Ad A M Masclee ${ }^{1}$ and José M Conchillo ${ }^{1 *}$ \\ Departments of ${ }^{1}$ Gastroenterology and Hepatology, and ${ }^{2}$ General Surgery, Maastricht University Medical Center, Maastricht, \\ The Netherlands
}

Electrical stimulation therapy (EST) of the lower esophageal sphincter is a relatively new technique for the treatment of gastroesophageal reflux disease (GERD) that may address the need of GERD patients, unsatisfied with acid suppressive medication and concerned with the potential risks of surgical fundoplication. In this paper we review available data about EST for GERD, including the development of the technique, implant procedure, safety and results from open-label trials. Two short-term temporary stimulation and long-term open-label human trials each were initiated to investigate the safety and efficacy of EST for the treatment of GERD and currently up to 2 years follow-up results are available. The results of EST are promising as the open-label studies have shown that EST is a safe technique with a significant improvement in both subjective outcomes of symptoms and objective outcomes of esophageal acid exposure in patients with GERD. However, long-term data from larger number of patients and a sham-controlled trial are required before EST can be conclusively advised as a viable treatment option for GERD patients.

(J Neurogastroenterol Motil 2014;20:287-293)

\section{Key Words}

Electrical stimulation therapy; Esophageal sphincter, lower; Gastroesophageal reflux disease

\section{Introduction}

Gastroesophageal reflux disease (GERD) is a highly prevalent disorder affecting approximately $20 \%$ of the Western population. ${ }^{1}$ Furthermore in Asia, where the prevalence of GERD is traditionally low, several reports suggest a rapid increase in prevalence over the last several years. ${ }^{2-4}$ The primary op- tion for the treatment of GERD is acid suppressive therapy, which has been shown to be effective in reducing symptoms and healing esophagitis. ${ }^{5,6}$ A large group of GERD patients however attend surgical and gastroenterology clinics for alternative treatment options as they have persistent troublesome GERD symptoms despite of optimal acid suppression or are unwilling to undergo lifelong medical treatment. ${ }^{7}$ Laparoscopic anti-reflux surgery (LARS) offers an established alternative treatment option to

Received: December 26, 2013 Revised: February 15, 2014 Accepted: February 23, 2014

(c) This is an Open Access article distributed under the terms of the Creative Commons Attribution Non-Commercial License (http://creativecommons. org/licenses/by-nc/3.0) which permits unrestricted non-commercial use, distribution, and reproduction in any medium, provided the original work is properly cited.

*Correspondence: José M Conchillo, MD, PhD

Department of Gastroenterology and Hepatology, Maastricht University Medical Center, Postbox 5800, 6202 AZ Maastricht, The Netherlands

Tel: +31-43-387-5021, Fax: +31-43-387-5006, E-mail: j.conchillo@mumc.nl

Financial support: None.

Conflicts of interest: None.

Author contributions: Nicolaas F Rinsma, analysis and interpretation of data, and drafting of the manuscript; Nicole D Bouvy and Ad A M Nasclee, critical revision of the manuscript for important intellectual content; José M Conchillo, analysis and interpretation of data, drafting and critical revision of the manuscript for important intellectual content and final approval. 
medical therapy, reducing both heartburn and regurgitation effectively. ${ }^{8}$ Many patients are however reluctant to this procedure, since new-onset dysphagia, gas-bloating, inability to vomit and/or belch are expected complications after LARS. ${ }^{9,10}$ Electrical stimulation of the lower esophageal sphincter (LES) is a relatively new technique for the treatment of GERD that may address the need of a large subgroup of GERD patients, unsatisfied with medical therapy and concerned with the potential risks of LARS. The aim of electrical stimulation therapy (EST) is to enhance the barrier function of the LES in order to obtain reflux control, while preserving normal LES function and esophagogastric junction (EGJ) anatomy. In this review we present available data about EST for GERD, including a short overview of the development of the technique, a description of the implant procedure, and safety aspects and results of the performed open-label studies. $^{11-13}$

\section{Development of Electrical Stimulation Therapy for Gastroesophageal Reflux Disease}

The first studies on electrical stimulation of the LES for the treatment of GERD were performed in canine models. Ellia et $\mathrm{al}^{14}$ reported that electric stimulation $(20 \mathrm{~Hz}$, pulse width of $3 \mathrm{msec}$ ) with 2 pairs of electrodes caused a prolonged contraction of the sphincter complex, which was able to prevent gastroesophageal reflux in dogs with surgically-induced EGJ incompetence. In another animal study which investigated pulse parameters in more detail, it was found that only low frequency stimulation (6 cycles per minute, pulse width of $375 \mathrm{msec}$ ) caused a prolonged increase in LES pressure. ${ }^{15}$ The effect of stimulation was sustained even after the stimulation had stopped, while LES relaxations during swallows were unaffected. High frequency stimulation $(20 \mathrm{~Hz}$, pulse width of $200 \mu \mathrm{sec}$ ) by endoscopically placed microstimulators into the esophageal muscle layer showed similar results, but only after exceeding a $8 \mathrm{~mA}$ threshold. ${ }^{16}$ Rodriguez et $\mathrm{al}^{17}$ were the first to investigate the effects of high $(20 \mathrm{~Hz}$, pulse width of $200 \mu \mathrm{sec}$ ) and low (6 cycles per minute, pulse width of $375 \mathrm{msec}$ ) frequency electrical stimulation on LES in GERD patients. Electrical stimulation with both high and low frequency caused an increase in LES pressure without affecting LES relaxation and LES residual pressure during swallowing. High frequency stimulation was determined to be a better option as it uses less energy and increases battery life. Similar to the outcomes of the animal studies, the effect of stimulation was sustained even af- ter the stimulation was stopped. These results were replicated by Banerjee et $\mathrm{al}^{18}$ using temporary stimulation leads implanted during endoscopy.

\section{Technique}

The LES-stimulation system (EndoStim BV, the Hague, The Netherlands) consists of 3 components: an implantable pulse generator (IPG), a bipolar lead with 2 stitch electrodes and an external programmer (Fig. 1). Electrical stimulation to the LES is generated by the IPG, sending electrical pulses of $5 \mathrm{~mA}$ at a rate of $20 \mathrm{~Hz}$ via the bipolar lead to the 2 stitch electrodes, implanted in the LES muscle. The IPG contains a non-rechargeable battery with a lifetime of 10-15 years, which is inserted in a subcutaneous pocket in the anterior abdominal wall (Fig. 2). The external programmer is used to modify parameters of the stimulation and to read therapy statistics. EST is delivered 8-12 times a day for 30 minutes per session. Temporary LES-stimulation studies had shown a sustained effect of EST on LES pressure that persisted long after stimulation was stopped, enabling the intermittent stimulation of the LES. ${ }^{17}$

\section{Implant Procedure}

Implantation of the IPG and bipolar lead is performed using

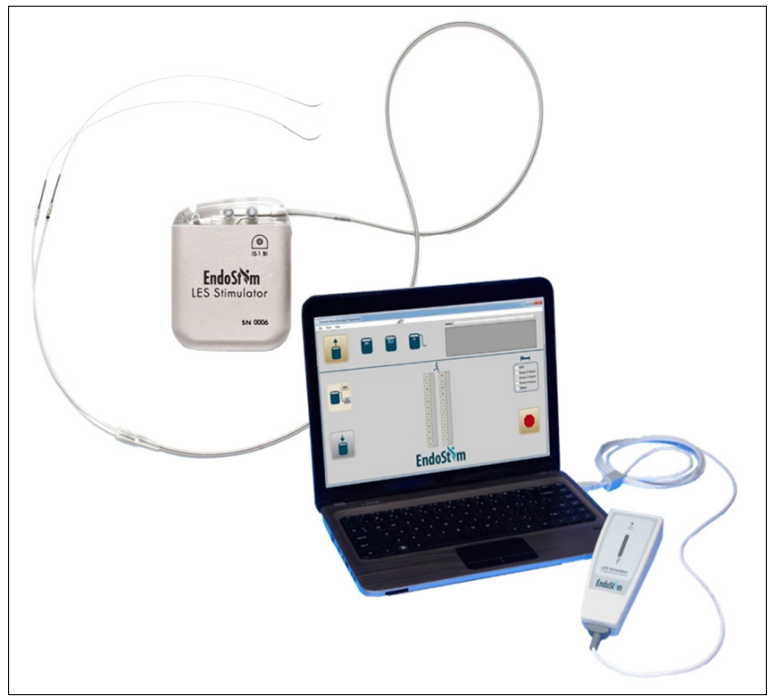

Figure 1. Lower esophageal sphincter (LES) stimulation system (EndoStim) consists of an implantable pulse generator (IPG), bipolar electrodes implanted in the LES and the leads connected to the IPG and an external programmer. 


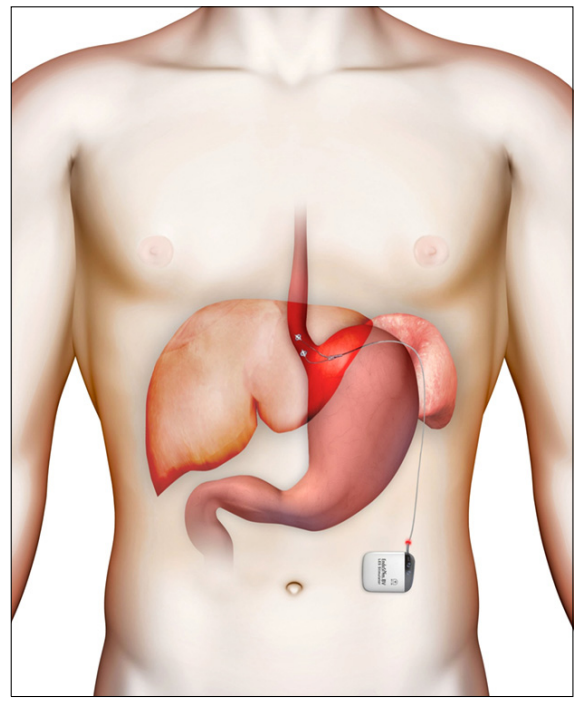

Figure 2. EndoStim implantable pulse generator implant is located in a subcutaneous pocket in the anterior abdominal wall and the bipolar electrodes are implanted in the lower esophageal sphincter muscle.

standard laparoscopic techniques, which were described previously in detail. ${ }^{11}$ In short, after trocar placement, dissection of fat tissue is performed to expose the muscle wall of the distal esophagus. In the presence of a small hiatal hernia, repair by caudal retraction of the esophagus and tightening of the hiatus by standard surgical procedure is indicated. The bifurcated lead is then introduced into the abdominal cavity and using a guiding needle, the electrodes at the proximal end of the lead are inserted and secured into the esophageal muscle wall (Fig. 2) Upper gastrointestinal endoscopy is performed to identify the Z-line by transillumination and to avoid perforation during electrode placement. The distal end of the bipolar lead is retracted through the abdominal wall and connected to the IPG. After a functionality test of the whole implanted system by the operator, the IPG is then placed into a subcutaneous pocket $3-5 \mathrm{~cm}$ below the left costal margin. After the anesthetics have worn off, the IPG is programmed for electrical stimulation therapy. Patients usually stay over in the hospital for one night for observation and are advised to wear an elastic compression bandage over the subcutaneous pocket and the IPG for 10-14 days to prevent formation of a seroma.

\section{Safety}

The results of the current studies demonstrate the safety of LES stimulation with an excellent side effect profile. In the 2 clinical trials so far 3 serious adverse events (SAE) were reported. One SAE was procedure related: a trocar perforation was identified on post-operative day one and was successfully repaired laparoscopically. The lead and the IPG were explanted prophylactically and the patient recovered well. The other 2 SAEs (thyroid surgery and cardiac arrhythmia-atrioventricular [AV] nodal reentrant tachycardia successfully treated with AV nodal ablation) were not device or therapy related. Of the remaining 77 non-SAE, 26 events had probable or definite relation to the procedure or the device (implant site pain, localized infection and post-operative nausea). Mild transient dysphagia was reported in 2 patients that underwent hiatal hernia repair which resolved spontaneously without intervention. No GI side effects such as diarrhea, bloating and inability to belch were reported and no cardiac side effects with LES-EST were observed.

\section{Efficacy}

Two open-label clinical studies investigating the efficacy and safety of EST of the LES using the EndoStim LES stimulation system were initiated and showed promising efficacy with results up to 24 months of follow-up. ${ }^{11-13}$

In the first open-label, single center trial performed in Chile, 25 GERD patients ( 14 men, mean age 52 years) with chronic (> 6 months) GERD symptoms, such as heartburn, regurgitation or retrosternal pain were included. GERD was well documented by 24-hour pH-monitoring and upper GI endoscopy, showing pathologic acid exposure time $(\mathrm{pH}<4.0$ during $>5.0 \%$ of time) and/or esophagitis. Main exclusion criteria were the presence of hiatal hernia $>3 \mathrm{~cm}$, esophagitis $>$ Los Angeles grade C, long-segment Barrett's esophagus or dysplasia. GERD symptoms were evaluated with GERD-health-related quality of life (HRQL), a disease-specific quality of life questionnaire, at baseline (on and off proton pump inhibitor [PPI] therapy) and during follow-up of electrical stimulation therapy. ${ }^{19}$ Furthermore, endoscopy, 24-hour pH-monitoring and high-resolution manometry were performed for secondary study endpoints. One patient withdrew consent 2 weeks after implantation due to anxiety related to the device and multiple follow-up tests required by the protocol so the IPG was explanted despite a favorable initial symptom response.

The first results of this trial showed improvement of symptom scores soon after activation of the device and at 6 months median (interquartile range [IQR]) GERD-HRQL symptom score was $2(0-4)$, which was significantly better than the median 
Table. Outcomes of Lower Esophageal Sphincter-Electrical Stimulation Therapy on Gastroesophageal Reflux Disease-Health-Related Quality of Life Symptom Scores, 24-hour pH-monitoring and Manometry of the First Open-label Human Trial at 12 Months Compared to Baseline $(\mathrm{n}=24)$ (Adapted from Rodriguez et $\mathrm{al}^{12}$ )

\begin{tabular}{lccc}
\hline & Baseline (Off PPI) & 12 months & $P$-value \\
\hline GERD-HRQL symptom score & $23.5(21.00-25.75)$ & $2.0(0.0-3.0)$ & $<0.010$ \\
Acid exposure time $(\%$ of time with $\mathrm{pH}<4.0)$ & $10.1(7.7-15.5)$ & $3.3(1.8-6.9)$ & $<0.001$ \\
Patients with abnormal AET & $24 / 24(100)$ & $8 / 22(39)$ & $<0.001$ \\
$\quad(\mathrm{n}[\%])(\mathrm{pH}<4.0$ during $>4.0 \%$ of time) & & & $\mathrm{N}$ \\
LES end-expiratory pressure $(\mathrm{mmHg})$ & $8.7(6.3-10.0)$ & $23.0(16.0-26.8)$ & $\mathrm{N} / \mathrm{A}$ \\
LES mean pressure $(\mathrm{mmHg})$ & $17.6(15.2-21.0)$ & $8.0(4.0-11.0)$ & $\mathrm{N} / \mathrm{A}$
\end{tabular}

PPI, proton pump inhibitor; GERD, gastroesophageal reflux disease; HRQL, health-related quality of life; AET, acid exposure time; LES, lower esophageal sphincter; N/A, not available as different manometry systems were used at baseline and 12-month follow-up.

All data are presented as median (IQR) unless indicated otherwise.

baseline scores both on PPI (9 [6-10], $P<0.001)$ and off PPI therapy (24 [21-26]). Twenty-one of 24 (88\%) patients were able to completely stop PPI usage. Acid exposure time during 24-hour pH-monitoring improved from median of $10.1 \%$ at baseline to a median of $5.1 \%$ after 6 months $(P<0.001) .{ }^{11}$

This study was initially designed with a 6-month follow-up period, but was extended to 2 years in order to collect additional long-term data. Twenty-three out of the initial 25 patients entered the extension phase. One patient did not enroll into the extension phase of the trial since he was planning to undergo a Roux-en-Y gastric bypass procedure for uncontrolled Type 2 diabetes. Twenty-three patients completed one year follow-up and the results were recently published. ${ }^{12}$ Median (IQR) GERDHRQL symptom score remained low at 12 months (2 [0-3], compared to baseline off PPI $[P<0.001]$, and baseline on PPI $[P=0.002])$ (Table). Median acid exposure time decreased even further to $3.3 \%$ from baseline $(P<0.001)$ and 14 of 22 $(64 \%)$ patients showed normal distal esophageal acid exposure time ( $\mathrm{pH}<4.0$ during $<4 \%$ of time). The trial also showed normalization of proximal esophageal acid exposure in subset of patients with proximal reflux at baseline. High-resolution manometry showed an increase of end expiratory and mean LES pressure, whereas esophageal body function and LES relaxation were unaffected (Fig. 3). New-onset dysphagia or sensation of the electrical stimulation was not reported. Currently, 21 patients have completed 24-month follow-up and the favorable outcomes of EST both on symptom scores and acid exposure time prevail over time. Seventy-one percent of patients showed either a normalized or at least $50 \%$ reduction in acid exposure time.

The second ongoing open-label multicenter study was initiated at university hospitals in the Netherlands, but patients were
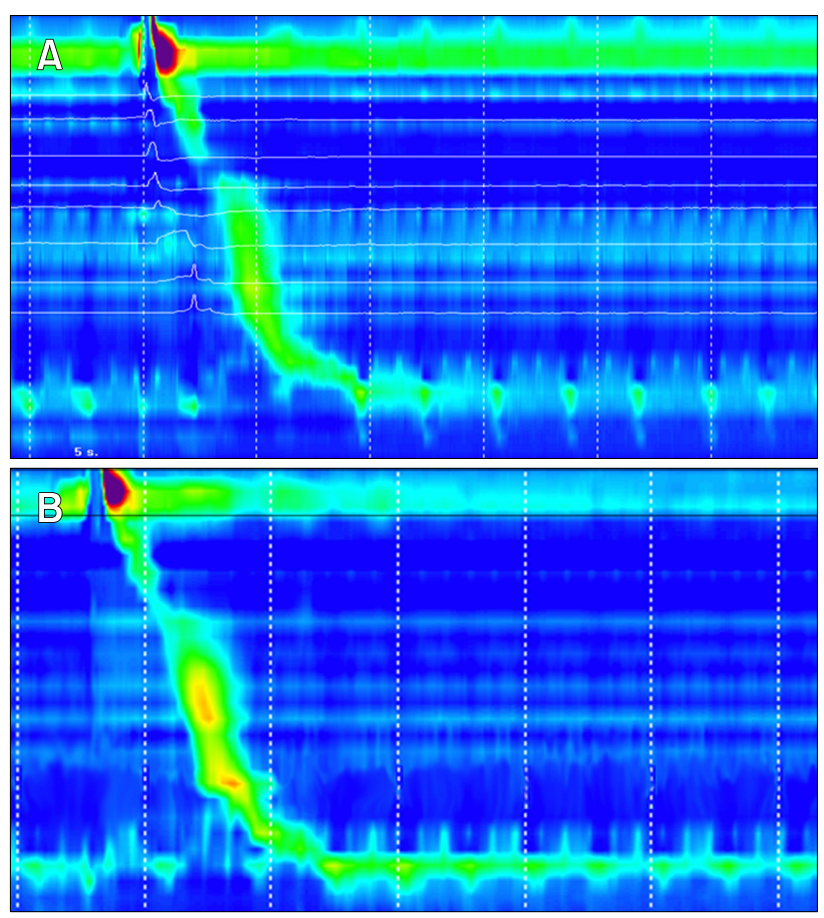

Figure 3. Effective primary peristalsis after a wet swallow $(5 \mathrm{~mL}$ of water) with complete relaxation of the lower esophageal sphincter (LES) seen on high-resolution manometry during electrical stimulation therapy. LES-end expiratory pressure was $12 \mathrm{mmHg}$ at baseline (A) which increased to $22 \mathrm{mmHg}$ after 3 months of LES-electrical stimulation therapy (B).

also recruited from New Zealand, India, Hong Kong and Chile. With similar inclusion and exclusion criteria as in the first openlabel trial, 24 patients (14 men, median age 51) were included. Currently, 18 patients have completed 3-month follow-up and 14 patients 6-month follow-up. Interim results showed a positive ef- 


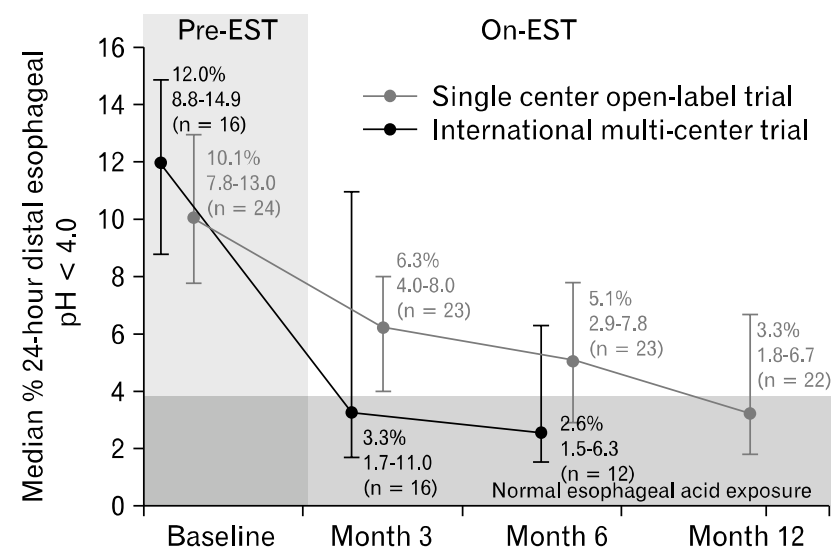

Figure 4. Improvement of acid exposure time in the 2 open-label trials. Pre-EST, before electrical stimulation therapy; On-EST, during electrical stimulation therapy.

fect on symptoms: GERD-HRQL median (IQR) score was 4 (2-11) at 3 months and 5 (4-9) at 6 months, respectively with $P$ $<0.001$ and $P<0.01$ when compared to baseline score off PPI (31 [25-37]). Furthermore, acid exposure time decreased from $11.3 \%(9.0-15.5 \%)$ at baseline to $3.3 \%(2.5-9.1 \%)$ at 3 months and to $2.6 \%(1.8-5.4 \%$ ) at 6 months (Fig. 4$) .{ }^{13}$ Completion of the data up to 24-month follow-up is awaited.

\section{Discussion}

At present, available data on EST of the LES indicate that EST may be an effective and safe treatment option for GERD patients searching for an alternative to medical therapy or conventional LARS.

The pathophysiology of GERD is multi-factorial; several factors can lead to the prolonged exposure of the esophageal epithelium to gastric acid and cause the perception of heartburn and/or regurgitation. ${ }^{20,21}$ The primary mechanism to prevent gastroesophageal reflux however is a competent barrier function of the EGJ, composed of the LES and the crural diaphragm. ${ }^{22}$ In GERD patients, the barrier function of the EGJ is impaired, allowing reflux to occur more easily than in healthy subjects. ${ }^{23}$ Acid suppressive therapy does not influence the function of the EGJ and symptoms due to reflux of non-acid gastric contents can persist. $^{24,25}$ LARS focuses on reinforcement of the EGJ by wrapping the gastric fundus around the distal esophagus. The procedure is effective in reducing gastroesophageal reflux, but the mechanical effects of the wrap can exceed the primary goal and lead to new onset dysphagia and inability to belch. ${ }^{26}$ Additional prob- lems such as diarrhea and gas-bloating have been reported. ${ }^{9,27,28}$ Due to the risks of postoperative complications, many patients dissatisfied with PPI therapy are reluctant to undergo this procedure.

Several alternative options for the treatment of GERD have been introduced during the last 2 decades. Less-invasive endoscopic suturing devices as the Endocinch (Bard Endoscopic Technologies, Nurray Hill, NJ, USA), Plicator (NDO Surgical Inc., Mansfield, MA, USA) and the EsophyX (EndoGastric Solutions, Inc., Silver Spring, MD, USA) have tried to mimic the effects of conventional anti-reflux surgery by the creation of gastroesophageal plications. ${ }^{29-31}$ Although early improvements in symptoms and medication use were reported in several trials, clinical efficacy or durability to the level of conventional anti-reflux surgery have not been met. ${ }^{32}$ The Enteryx procedure aimed to increase sphincter competence by injection of sponge-like material into the LES. A sham-controlled study showed a moderate effect on symptoms and PPI use, however the product was withdrawn after several reports of severe adverse events related to the injection of the substance. ${ }^{32,33}$ The Stretta device (Curon Medical, Sunnyvale, CA, USA) delivers radiofrequency energy to the LES muscle, inducing fibrosis of the LES muscle and thereby augmenting its function. Although a mild subjective improvement was shown in a sham-controlled trial, it failed to improve objective reflux parameters. ${ }^{34}$ All these therapies were unable to demonstrate durable and clinically meaningful improvement in distal esophageal acid exposure. Another recent alternative is the magnetic sphincter, a bracelet of magnetic beads (LINX-device; Torax Medical, St. Paul, MN, USA), which is laparoscopically placed around the LES. A recently published study showed encouraging results on acid exposure time and symptom improvement; however the high frequency of dysphagia requiring dilation is similar to that of conventional anti-reflux surgery and in a fraction of patients the device was removed because of SAE. ${ }^{35}$ So far, no randomized controlled trials are available to fully assess the effect of the LINX-device.

The current results of EST are promising as the 2 open-label studies have shown that EST is a safe technique with significant improvement in both subjective outcomes and objective parameters of GERD. LES stimulation significantly enhanced LES pressure, reduced esophageal acid exposure time and improved GERD symptom scores.

Interestingly, the effect of EST on acid exposure time increased during follow-up in the first open-label trial and also the preliminary results of the multicenter trial show a comparable im- 
provement on acid exposure over time (Fig. 4). These findings could be due to the possibility of EST to non-invasively change the settings of the IPG to optimize the anti-reflux effect. However, in the current trials adjustments to the IPG settings were limited per protocol and stimulation parameters as pulse width, pulse frequency and pulse shape were similar for all patients. Future trials are needed to reveal if tailored-fit electrical stimulation therapy, customized to the patients' individual needs, may even further improve the anti-reflux effect of EST.

The mechanisms underlying the anti-reflux effect of EST have not been studied in detail. The observed increase in LES pressure can contribute to the anti-reflux effect of the procedure; however other mechanisms as reduction of the number of transient LES relaxations and changes in gastric motility may also play a role and need to be studied.

The implant procedure is quite simple to perform for general laparoscopic surgeons and can be easily standardized for uniform performance. In comparison to laparoscopic fundoplication, there seems to be a shorter learning curve for the implantation procedure of EST. Therefore, it is no surprise that results from the multicenter trial in medical centers that performed only a few implantations are similar to the outcomes of the experienced single center trial. Furthermore in contrast to laparoscopic fundoplication, the EGJ anatomy is unaltered during the implant procedure, making EST easily reversible by removal of the electrodes and IPG.

Future prospects for LES-EST include targeting a wider range of GERD patients. Unlike traditional anti-reflux surgery or procedures, LES-EST does not adversely affect esophageal body function or LES relaxation and hence could be an important therapeutic alternative for GERD patients with esophageal dysmotility that are not adequately addressed by medical management. Furthermore, post-sleeve gastrectomy reflux is a new and rising subgroup of GERD patients that are inadequately treated with medication and are not a candidate for traditional anti-reflux surgery. ${ }^{36}$ These patients could be addressed with LES-EST and a pilot study will be started in our center to evaluate the effect of EST in patients with post-sleeve gastrectomy GERD. Also improvement in the system's components is expected in the future. Reduction of the battery size could improve the cosmetic effect of the procedure and limit pocket related symptoms. Ultimately, endoscopic implantation of rechargeable electronic microstimulators would be an ideal anti-reflux procedure; this is however not expected in the very near future.

Some points need to be taken into consideration when inter- preting the data published so far. The results of the multicenter trial are preliminary and long-term follow-up data need to be awaited. Furthermore, study participants represent a subgroup of GERD patients with only small hiatus hernia and no severe esophagitis or Barrett's esophagus. Whether GERD patients with greater anatomical defects or more severe disease can benefit from EST is unknown, but the combination of hiatal hernia repair with EST might offer an alternative to LARS. Finally, the available data are obtained during non-randomized controlled studies. In order to further establish the effect of EST on GERD, a randomized sham-controlled trial should be performed in the near future. The technique of electrical stimulation of the LES is especially suitable for a sham-controlled study, as the device can be easily activated and turned-off.

In conclusion, current results suggest that EST is a safe and effective therapeutic alternative that may fill the gap between PPI therapy and conventional anti-reflux surgery. More long-term data and controlled trials are required before EST can be considered as an alternative for the treatment of GERD.

\section{References}

1. El-Serag HB, Sweet S, Winchester CC, Dent J. Update on the epidemiology of gastro-oesophageal reflux disease: a systematic review. Gut 2014;63:871-880.

2. Lim SL, Goh WT, Lee JM, Ng TP, Ho KY; Community Medicine GI Study Group. Changing prevalence of gastroesophageal reflux with changing time: longitudinal study in an Asian population. J Gastroenterol Hepatol 2005;20:995-1001.

3. Ho KY, Chan YH, Kang JY. Increasing trend of reflux esophagitis and decreasing trend of Helicobacter pylori infection in patients from a multiethnic Asian country. Am J Gastroenterol 2005;100:19231928.

4. Jung HK. Epidemiology of gastroesophageal reflux disease in Asia: a systematic review. J Neurogastroenterol Motil 2011;17:14-27.

5. Khan M, Santana J, Donnellan C, Preston C, Moayyedi P. Medical treatments in the short term management of reflux oesophagitis. Cochrane Database Syst Rev 2007;2:CD003244.

6. Kahrilas PJ, Shaheen NJ, Vaezi MF; American Gastroenterological Association Institute; Clinical Practice and Quality Management Committee. American Gastroenterological Association Institute technical review on the management of gastroesophageal reflux disease. Gastroenterology 2008;135:1392-1413, e5.

7. Fass R, Sifrim D. Management of heartburn not responding to proton pump inhibitors. Gut 2009;58:295-309.

8. Galmiche JP, Hatlebakk J, Attwood S, et al. Laparoscopic antireflux surgery vs esomeprazole treatment for chronic GERD: the LOTUS randomized clinical trial. JAMA 2011;305:1969-1977.

9. Straathof JW, Ringers J, Lamers CB, Masclee AA. Provocation of transient lower esophageal sphincter relaxations by gastric distension with air. Am J Gastroenterol 2001;96:2317-2323. 
10. Broeders JA, Mauritz FA, Ahmed Ali U, et al. Systematic review and meta-analysis of laparoscopic Nissen (posterior total) versus Toupet (posterior partial) fundoplication for gastro-oesophageal reflux disease. Br J Surg 2010;97:1318-1330.

11. Rodriguez L, Rodriguez P, Gómez B, et al. Electrical stimulation therapy of the lower esophageal sphincter is successful in treating GERD: final results of open-label prospective trial. Surg Endosc 2013;27:1083-1092.

12. Rodriguez L, Rodriguez P, Gómez B, et al. Long-term results of electrical stimulation of the lower esophageal sphincter for the treatment of gastroesophageal reflux disease. Endoscopy 2013;45:595604.

13. Siersema PD, Smout AJ, Conchillo JM, et al. Electrical stimulation therapy (EST) of the lower esophageal sphincter (LES) - an effective therapy for refractory GERD - interim results of an international multicenter trial. United European Gastroenterol J 2013;1(suppl 1):A411.

14. Ellis F, Berne TV, Settevig K. The prevention of experimentally induced reflux by electrical stimulation of the distal esophagus. Am J Surg 1968;115:482-487.

15. Sanmiguel CP, Hagiike M, Mintchev MP, et al. Effect of electrical stimulation of the LES on LES pressure in a canine model. Am J Physiol Gastrointest Liver Physiol 2008;295:G389-G394.

16. Clarke JO, Jagannath SB, Kalloo AN, Long VR, Beitler DM, Kantsevoy SV. An endoscopically implantable device stimulates the lower esophageal sphincter on demand by remote control: a study using a canine model. Endoscopy 2007;39:72-76.

17. Rodriguez L, Rodriguez P, Neto MG, et al. Short-term electrical stimulation of the lower esophageal sphincter increases sphincter pressure in patients with gastroesophageal reflux disease. Neurogastroenterol Motil 2012;24:446-450.

18. Banerjee R, Pratap N, Kalpala R, Nageshwar Reddy D. Effect of electrical stimulation of the lower esophageal sphincter using endoscopically implanted temporary stimulation leads in patients with reflux disease. Surg Endosc 2014;28:1003-1009.

19. Velanovich $V$. The development of the GERD-HRQL symptom severity instrument. Dis Esophagus 2007;20:130-134.

20. Bredenoord AJ. Mechanisms of reflux perception in gastroesophageal reflux disease: a review. Am J Gastroenterol 2012;107:815 .

21. Farré R. Pathophysiology of gastro-esophageal reflux disease: a role for mucosa integrity? Neurogastroenterol Motil 2013;25:783-799.

22. Mittal RK, Balaban DH. The esophagogastric junction. N Engl J Med 1997;336:924-932.

23. Pandolfino JE, Shi G, Trueworthy B, Kahrilas PJ. Esophagogastric junction opening during relaxation distinguishes nonhernia reflux patients, hernia patients, and normal subjects. Gastroenterology 2003; 125:1018-1024.
24. Zerbib F, Duriez A, Roman S, Capdepont M, Mion F. Determinants of gastro-oesophageal reflux perception in patients with persistent symptoms despite proton pump inhibitors. Gut 2008;57:156160.

25. Mainie I, Tutuian R, Shay S, et al. Acid and non-acid reflux in patients with persistent symptoms despite acid suppressive therapy: a multicentre study using combined ambulatory impedance- $\mathrm{pH}$ monitoring. Gut 2006;55:1398-1402.

26. Pandolfino JE, Curry J, Shi G, Joehl RJ, Brasseur JG, Kahrilas PJ. Restoration of normal distensive characteristics of the esophagogastric junction after fundoplication. Ann Surg 2005;242:43-48.

27. Broeders JA, Bredenoord AJ, Hazebroek EJ, Broeders IA, Gooszen HG, Smout AJ. Effects of anti-reflux surgery on weakly acidic reflux and belching. Gut 2011;60:435-441.

28. Marjoux S, Roman S, Juget-Pietu F, et al. Impaired postoperative EGJ relaxation as a determinant of post laparoscopic fundoplication dysphagia: a study with high-resolution manometry before and after surgery. Surg Endosc 2012;26:3642-3649.

29. Schwartz MP, Wellink H, Gooszen HG, Conchillo JM, Samsom M, Smout AJ. Endoscopic gastroplication for the treatment of gastro-oesophageal reflux disease: a randomised, sham-controlled trial. Gut 2007;56:20-28.

30. von Renteln D, Schiefke I, Fuchs KH, et al. Endoscopic full-thickness plication for the treatment of GERD by application of multiple Plicator implants: a multicenter study (with video). Gastrointest Endosc 2008;68:833-844.

31. Bell RC, Mavrelis PG, Barnes WE, et al. A prospective multicenter registry of patients with chronic gastroesophageal reflux disease receiving transoral incisionless fundoplication. J Am Coll Surg 2012; 215:794-809.

32. Pandolfino JE, Krishnan K. Do endoscopic antireflux procedures fit in the current treatment paradigm of gastroesophageal reflux disease? Clin Gastroenterol Hepatol 2014;12:544-554.

33. Devière J, Costamagna G, Neuhaus $H$, et al. Nonresorbable copolymer implantation for gastroesophageal reflux disease: a randomized sham-controlled multicenter trial. Gastroenterology 2005;128:532540.

34. Arts J, Bisschops R, Blondeau K, et al. A double-blind sham-controlled study of the effect of radiofrequency energy on symptoms and distensibility of the gastro-esophageal junction in GERD. Am J Gastroenterol 2012;107:222-230.

35. Ganz RA, Peters JH, Horgan S. Esophageal sphincter device for gastroesophageal reflux disease. N Engl J Med 2013;368:20392040.

36. Howard DD, Caban AM, Cendan JC, Ben-David K. Gastroesophageal reflux after sleeve gastrectomy in morbidly obese patients. Surg Obes Relat Dis 2011;7:709-713. 